\title{
1 Safety assessment of immune-mediated adverse reactions to novel food proteins
}

2

$$
\text { Antonio Fernandez }{ }^{1 *} \text {, E. N. Clare Mills², Frits Koning }{ }^{3} \text {, F. Javier Moreno }{ }^{4}
$$

3 1. European Food Safety Authority (EFSA), via Carlo Magno 1A, 43021 Parma, Italy.

4 2. Division of Infection, Immunity and Respiratory Medicine, School of Biological Sciences, Manchester Institute of

5 Biotechnology, Manchester Academic Health Sciences Centre, The University of Manchester, Manchester, UK.

6 3. Department of Immunohematology and Blood Transfusion, Leiden University Medical Centre, Leiden 2333 ZA,

7 The Netherlands.

8 4. Instituto de Investigación en Ciencias de la Alimentación, CIAL (CSIC-UAM), C/ Nicolás Cabrera, 9, Campus de la

9 Universidad Autónoma de Madrid, 28049 Madrid, Spain.

10 *Correspondence: antonio.fernandezdumont@efsa.europa.eu

11 FJM: ORCID iD: 0000-0002-7637-9542 ; Twitter account: @CIAL_CSIC_UAM

12 CM: ORCID iD: 0000-0001-7433-1740 


\section{Abstract}

14 Current international guidelines for the risk assessment of biotechnology-derived

15 foods date back to 2003. We present new strategies and directions for assessing

16 immune adverse reactions to novel food proteins. Understanding genetic factors

17 involved in food allergy and the role of the gastrointestinal tract will streamline risk

18 assessment strategies.

19 Keywords: risk assessment, in vitro digestion models, novel proteins, HLA genotype,

20 immune-mediated, celiac disease. 
The risk assessment (RA) of novel proteins is critical for food safety authorities because proteins can cause immune-mediated adverse reactions in susceptible individuals which can sometimes be life-threatening. Consequently, before new products can be placed on the market, a safety assessment is performed.

Currently, the mechanisms underlying immune-mediated adverse reactions are poorly understood, making it challenging to predict whether a novel protein has the potential to cause such reactions. A cumulative body of evidence, in a so called weightof-evidence approach, is used to reduce uncertainty and enhance the reliability of predictions [1, 2]. Overall, the RA strategy followed for the safety assessment of proteins is taken from the classical RA of "chemicals". However, such strategy has important limitations when applied to proteins.

There is evidence that gastrointestinal (GI) digestion can affect the immunogenicity of dietary proteins in relation to both IgE- and non-IgE-mediated adverse immune reactions to food $[3,4]$. Therefore, the fate of a novel protein in the GI tract environment and its presentation to the gut mucosal immune system are key considerations that can inform the RA process and should be taken into account in a weight-of-evidence approach. This requires a comprehensive characterization of a novel protein and its digestion products, and their capacity to interact with the mucosal immune system of the GI tract. Such data can provide a sound basis for the RA by identifying and characterizing the potential hazards presented by oral exposure to a novel protein. 

assessment of novel proteins focusing on IgE- and non-lgE-mediated adverse immune reactions to food. Through a critical view of past experience and considering the current scientific state-of-art, we propose the RA strategy recently developed for assessing the potential for a novel protein to cause celiac disease (CD) as a "gold standard" for food safety assessment of novel proteins in general.

Innovative safety assessments for immune-mediated adverse reactions to novel proteins in food

Risk assessment strategy for celiac disease

The mechanisms governing the pathogenesis of $C D$ are well characterized, and a pioneering strategy has been developed where specific in silico and in vitro approaches are proposed for assessing the risk that a protein could cause CD (Box 1) [5]. This is the first comprehensive scientific evidence-based strategy developed for the safety assessment of a novel protein's potential to cause CD. It has the capacity to predict a protein's ability to cause adverse effects with a higher degree of assurance compared to other traditional tools, such as those currently in use for the RA of IgEmediated adverse immune reactions. Furthermore, the proposed strategy represents a solid step forward in food safety, promoting public health and trust in the scientific community. Whilst a genetic predisposition towards atopy is known to play a critical role in the development of IgE-mediated adverse immune reactions to external agents, 
including food, genetic factors alone cannot explain why some individuals become allergic and others do not. Unlike $C D$, it is not currently feasible to define a reliable predictive approach for IgE-mediated food allergy because other environmental and developmental factors are involved. For instance, a recent study provided insights into the pathways modified by gene-environment interactions in a cohort of infants with challenge-confirmed egg allergy [6].

However, applying the predictive approach developed for proteins that can elicit $C D$ requires a better understanding of why some proteins and not others become allergens. This may be related to the role of the GI tract in exposure and of specific certain food proteins. Efforts to identify HLA types related to food allergy are beginning to provide insights. Thus, a series of informative and elegant GWASs, using a large cohort of populations from different geographical origins, has recently identified peanut- [7-9], shrimp- and peach-specific allergy loci [10] in the HLA-DR/DQ gene region. Possibly, these changes may relate to HLA alleles that predispose individuals to IgE-mediated food allergy to particular allergens by affecting antigen presentation events. This information could support the future development of more powerful tools for the RA of novel proteins with regards IgE-mediated food allergy.

In vitro digestion studies in risk assessment for adverse immune reactions The current approach to assess the safety of proteins follows clear logic: if the novel protein under assessment is extensively degraded in the GI tract and does not share significant structural similarities with known allergens, it is unlikely that the 
strategies rely on the classical pepsin-resistance test, which is known to have several inherent limitations as a predictor of both allergenicity and digestion [11]. Recent work considers how the classical pepsin-resistance test can be refined to better reflect physiological conditions and to provide more reliable knowledge about the fate of the novel protein in the Gl tract [5]. Briefly, the refined in vitro protein digestion test proposal considers: (1) a variety of gastric as well as intestinal conditions targeting different segments of the population such as infants and adults; (2) frames the readout requirements more clearly (SDS-PAGE and LC-MS $)$; (3) focuses on the persistence/transience and abundance of stable fragments; and (4) defines a cut-off value for peptides of 9 amino acids in length which is based on the minimum peptide length able to activate T-cells.

Current evidence [12] suggests that not only the intact protein but also stable fragments surviving Gl digestion should be evaluated when addressing immune adverse reactions to proteins. Nevertheless, surprisingly few studies have investigated the immunogenicity or sensitizing capacity of digested proteins [13]. Identifying relevant fragments (according to size, persistence and abundance), surviving in vitro GI digestion and capable of interacting with the immune system, is essential to develop a more comprehensive approach for protein safety assessment (Figure 1). The EFSA is currently testing the practicality of these refined conditions. ${ }^{i i}$ The in vitro GI digestion test that will result from the testing phase could become a fit-for-purpose tool providing more reliable information to the weight-of-evidence approach and the overall RA strategy. Furthermore, the development of advanced risk assessment tools, based on state-of-the-art knowledge (Figure 1), is needed to decipher the potential 
correlation between stable fragments resulting from GI digestion and their capacity to cause immune adverse reactions.

\section{Concluding remarks and future perspectives}

Assessing the safety of novel proteins in the context of immune-mediated adverse reactions challenges the current RA paradigm because of the limited knowledge of disease mechanisms. The relatively low prevalence of these conditions also means that a food protein can be innocuous for $99 \%$ of the human population and hazardous for the remaining $1 \%$ susceptible individuals, which poses additional scientific challenges. An exception to the limited knowledge is CD.

Considering the relevance of oral exposure in the RA of food proteins, proteinGI tract interactions are the key element to frame and build the most robust strategy. Here we highlight the crucially important fact that food protein fragments, which are stable to digestion, can be even more hazardous than the intact protein, as is the case for gluten. So far, the assessment process has mainly focused on the properties of the intact protein, even though it changes during its passage through the $\mathrm{Gl}$ tract. Considering recent advances in peptidomics, a comprehensive characterization of the stable digestion fragments and a better understanding of their interactions with the immune system are needed to gain new insights on the potential immunogenicity and, hence, allergenicity of proteins. Nevertheless, gaps in our knowledge remain and present a focus for future research efforts.

For instance, the most suitable in vitro digestion models and items to test, which could better elucidate the interaction between proteins/fragments and the $\mathrm{Gl}$ 
tract/immune system, are yet to be unraveled. Also, the criteria to identify digestion fragments as relevant for RA (based on abundance, persistence and size) need to be

137 verified. Furthermore, given the lack of knowledge about the regulation of allergenspecific IgE at the cellular level, research projects exploring the association between

139 IgE-food allergy and genetic factors, such as HLA type and polymorphisms, are pivotal 140 to explaining the pathogenesis of IgE-mediated reactions. Finally, the current 141 understanding of the pathogenesis of non-IgE-mediated diseases other than CD is very 142 limited, and additional research to provide a reliable framework for risk assessment is needed.

Food safety assessment should rely on studies at the cutting edge of science, which implies continuous updates. The main principles of current international guidelines for products derived from biotechnology date back to 2003 [1]. New approaches are available, and there is sufficient scientific evidence that justifies further improvements of current international guidelines (e.g., for CD). We encourage the scientific community to work towards better understanding stable digestion fragments and their interactions with the immune system in the GI tract.

\section{Acknowledgements}

The authors wish to thank Claudia Paoletti for the inspiring comments. 
156 Antonio Fernandez is employed by the European Food Safety Authority (EFSA). The

157 positions and opinions presented in this article are those of the authors alone and do

158 not necessarily represent the views or scientific works of EFSA. The authors declare

159 that they have no conflict of interest.

160

161

Resources

162

'https://doi.org/10.1016/j.toxlet.2018.06.722

163

iihttps://ted.europa.eu/udl?uri=TED:NOTICE:289049-2017:TEXT:EN:HTML

164

165

\section{References}

166

1. Codex Alimentarius (2003-2009) Foods derived from modern biotechnology. Codex Alimentarius Commission, Joint FAO/WHO Food Standards Programme, Rome.

2. EFSA GMO Panel (EFSA Panel on Genetically Modified Organisms) (2011) Scientific opinion on guidance for risk assessment of food and feed from genetically modified plants. EFSA J. 9(5), 2150.

171

3. Pekar, J. et al. (2018) Stability of allergens. Mol. Immunol. 100, 14-20.

172

4. Pali-Schöll, I. et al. (2018) The effect of digestion and digestibility on allergenicity of

173 food. Nutrients 10, 1129.

174 5. EFSA GMO Panel (EFSA Panel on Genetically Modified Organisms) (2017). Guidance

175 on allergenicity assessment of genetically modified plants. EFSA J. 15(6), 4862. 
176 6. Martino, D. et al. (2018) Epigenetic dysregulation of naive CD4+ T-cell activation 177 genes in childhood food allergy. Nat. Commun. 9, 3308.

178 7. Hong, X. et al. (2015) Genome-wide association study identifies peanut allergy179 specific loci and evidence of epigenetic mediation in US children. Nat. Commun. 6, 1806304.

8. Martino, D.J. et al. (2017) Genome-wide association study of peanut allergy reproduces association with amino acid polymorphisms in HLA-DRB1. Clin. Exp. Allergy 47, 217-223.

9. Marenholz, I. et al. (2017) Genome-wide association study identifies the SERPINB gene cluster as a susceptibility locus for food allergy. Nat. Commun. 8, 1056.

10. Khor, S.-S. et al. (2018) Genome-wide association study of self-reported food reactions in Japanese identifies shrimp and peach specific loci in the HLA-DR/DQ gene region. Sci. Rep. 8, 1069.

11. Bøgh, K.L. and Madsen, C.B. (2016) Food Allergens: Is there a correlation between stability to digestion and allergenicity?. Crit. Rev. Food Sci. Nutr. 56, 1545-1567.

12. Bethune, M.T. and Khosla, C. (2008) Parallels between pathogens and gluten 192 peptides in celiac sprue. PLoS Pathog. 4(2), e34. rats. Clin. Exp. Allergy 39, 1611-1621. 297, 2275-2279. 

15. Sollid, L.M. et al. (2012) Nomenclature and listing of celiac disease relevant gluten

198 T-cell-epitopes restricted by HLA-DQ molecules. Immunogenetics 64, 455-460. 
201 Gluten (and gluten-like) proteins are of proline-rich nature, which confers them

202 stability to degradation by enzymes of the GI tract. Relatively long gluten fragments 203 are observed in the small intestine [14], which renders them available for interaction 204 with the mucosal immune system of the GI tract, contributing to their immunogenicity. Remarkably, it is via the action of endogenous GI proteases that immunotoxic gluten peptides are released to enact their harmful effects [12]. These fragments are described to be more toxic than the intact dietary gluten proteins themselves, providing novel insights for the development of RA strategies. Expression of certain HLA genotypes is a key risk factor for developing a disease condition that is driven by activated T-cells [15]. The current knowledge available on HLA-DQ structures and on

211 the binding recognition sites for T-cell activation, allows defining accurate strategies 212 for assessing the potential CD capacity of proteins. Based on these considerations, we 213 proposed a pioneer strategy for the RA of CD (Figure I).

214 Figure I. Stepwise approach for the risk assessment of celiac disease (adapted from $215[5])$. 
217 Figure 1. Criteria and methodological tools needed to identify gastrointestinal 218 digestion fragments as potential hazards for the risk assessment of immune-mediated 219 adverse reactions to novel proteins are described. Understanding what the fate of the 220 protein in the digestive tract is and how it interacts with the immune system are key elements to consider. Fragments resulting from the gastrointestinal digestion of novel

222 proteins will raise different levels of concern being the highest concern for those

223 fragments that are large, persistent and, in addition, abundant. The current state of 224 scientific knowledge in the pathogenesis of celiac disease has allowed the 225 development of a stepwise approach where the risk assessment process could be 226 stopped at the initial in silico step if no concerns are identified (Box 1). In the case of 227 IgE-mediated reactions, additional developments in the several steps of the safety 228 assessment (in silico, in vitro and in vivo) are desirable to provide more robust 229 information for the allergenicity assessment within the weight-of-evidence approach. 


\section{Ms. Ref. No.: TIBTECH-D-18-00289 “Safety assessment of immune-mediated adverse reactions to novel food proteins" Fernandez et al.}

Dear Dr. Pavlovich,

We would like to thank you and the reviewers for the thorough review of the manuscript. A number of interesting issues have been raised and the received inputs have been very helpful for improving the clarity of the manuscript. We have considered all comments and we have revised our manuscript accordingly. We are confident that the revised version of the manuscript has been improved. We respond below in detail to each of the editor and reviewer's comments. We hope that you will find our responses satisfactory to the raised comments.

\section{EDITORIAL COMMENTS:}

Title Page

1. We encourage our authors to list contact information such as ORCIDs, Twitter or social media handles, or links to lab websites, which will be linked to in the online version of the manuscript on the title page; feel free to add this information if you are interested.

RESPONSE: Additional information has been incorporated as requested.

Content

2. Each of the reviewers has good suggestions for improving the manuscript that nevertheless may be difficult to implement within the space constraints of this format. Reviewer \#2 in particular has a long list of thoughtful considerations for making the proposed approach more specific and detailed and emphasized in a separate set of comments that these are not mandatory but simply points for consideration. The reviewer further suggests a more detailed visual aid that traces the steps of the proposed process, which I strongly agree with; it could incorporate elements from the current Figure 1 but extend the information into a more visually intuitive process-like diagram. Then, the caption for that figure could also be expanded to provide some of the specifics that the reviewer suggests.

RESPONSE: The Figure 1 has been substantially modified in order to make it more informative and visually intuitive process-like diagram, as requested. In addition, new information based on the comments provided by the reviewer 2 (as the use of MHC-associated peptide proteomics 
(MAPPs) to identifying T cell epitopes) is now included. Accordingly, the caption has been also expanded to provide more details about the proposed risk assessment strategies for celiac disease and IgE-mediated adverse reactions.

3. The other reviewers also have good suggestions for improving the manuscript; please read these and revise the manuscript to the extent that it's possible while remaining within the word limit.

RESPONSE: The manuscript has been revised according to referees' comments but preserving the maximum word limit. Please, see below for further information.

Figures/Tables/Text Boxes

4. Please upload individual image files for each of your figures (including figures contained within Text Boxes) along with the revised manuscript. Please see our Author Instructions under the Figure Guidelines tab for more information about acceptable file types and resolution. If possible, please include both a finalized image (.jpg, .tif, or .pdf preferred) and an original file that the image was created from (for instance, .ppt or .ai) for each figure. Finally, please note that the typesetter will add the correct Figure 1 or Figure I label to the image, so please upload the finalized images and original files without these labels.

RESPONSE: Figure 1 and Box 1 have been uploaded and modified according to the Author Instructions.

5. Please attach the contents of Box 1 to the end of the manuscript rather than uploading the box as a separate file to avoid a delay in typesetting.

RESPONSE: Done.

6. Regarding the proposed expanded Figure 1, I'm happy to inform you that we offer complimentary professional illustration support in collaboration with the Elsevier Webshop; please email me prior to resubmission if you are interested in this service.

RESPONSE: If possible, we would appreciate very much a check of the updated Figure 1 and Figure I in order to know if the new versions are suitable for publication. 


\section{References and Length}

7. During revision, please keep the main text at no more than 1500 words. This limit does not include the Abstract, References, or any additional elements (Figures, Tables, Text Boxes). Please note that this limit is strict for this article format.

RESPONSE: As mentioned above, the main text of the revised version is within the limit.

8. Please note that the Abstract is strictly limited to 50 words.

RESPONSE: Abstract of the revised version contains 48 words.

9. Please ensure that the revised manuscript cites no more than 15 references. The reviewers suggest some additional references to cite, which I encourage you to review. If you think it is appropriate to add them, it would be necessary to remove an existing reference; I'd suggest one or more of references 12-15 as each of these is older and on similar topics.

RESPONSE: The revised manuscript cites 15 references. The reviewers' suggestions have been acknowledged and conveniently discussed in this document (please, see below).

Clarity and Accessibility

10. Finally, please review the changes I have suggested in the main text and make any modifications necessary if the intended scientific meaning has been altered. In particular, please note that Forum articles should be written in a more "journalistic" or scientifically informal style compared to review articles, so I have provided some suggestions to meet that style.

RESPONSE: We thank you for the proposed changes in the main text which have been accepted conferring the manuscript a more readable journalistic style.

\section{REVIEWER \#1:}

This article from EFSA and EFSA panel members, suggests a new approach to risk assessment of potential new allergens. The article rightly points out that it is hard to assess risk when routes of sensitisation are unknown. The article is well written and should be published after the minor points outlined below have been addressed. 
RESPONSE: We appreciate the positive comments on our manuscript.

The suggestions are based on an extension of the approach used in coeliac disease including two separate areas of development, namely genomics and in vitro digestion. This idea is not new but seems a logical approach. It is clear that we are still some way from being able to define the genetic risk at a population level or indeed for an individual but the idea of personalised risk as an extension of personalised nutrition is interesting. The author might speculate on how they envisage this approach working.

RESPONSE: The referee raises an interesting but challenging question. As we indicated in the manuscript, to gather knowledge on HLA phenotypes is key to understand the pathogenesis of immune mediated diseases. Nevertheless, by considering the large heterogeneity of proteins described as IgE allergens and given the lack of current knowledge on genomic regions involved in food allergy mediated by $I g E$, it is a chimera to expect that HLA phenotypes governing IgE disease could be unravelled for all known allergens. Therefore, it would be reasonable to reach a consensus to prioritise and complete the work on HLA phenotypes focusing on specific allergens. Moreover, as we also indicated in our manuscript, genetic factors alone cannot explain why some individuals become allergic and others do not, because other environmental and developmental factors are involved in IgE-mediated food allergy. Therefore, to envisage when the genetic risk at a population level could be well defined in IgE-mediated reactions and how this information can be used in the risk assessment process is rather challenging at this point in time.

The section on digestion should include information on which section of the population is being simulated. In the case of allergy, there is a marked difference in risk between adults and children and this needs to be reflected in the simulations used to assess the persistence of peptides that may be of risk to the different demographic groups.

RESPONSE: We fully agree with the referee's remark. In fact, the digestibility of the novel food proteins in specific segments of the population such as infants, adults or individuals with impaired digestive functions is considered in the refined in vitro protein digestion tests proposed by EFSA by using different gastric and intestinal conditions comprising different $\mathrm{pH}$ values and variable concentrations of digestive enzymes and biosurfactants based on collected in vivo data. In this context, and following referee's remark, a brief clarification has been 
included in the revised version of the manuscript by adding the following sentence: "Briefly, the refined in vitro protein digestion test proposal considers a variety of gastric as well as intestinal conditions targeting different segments of the population such as infants and adults;..."

\section{REVIEWER \#2:}

The manuscript categorized under "Forum" aims to provide a new, more modern strategy for the immunological risk assessment of foods that are produced using biotechnology. The authors correctly state that the international guidelines that are currently used date back to 2003 and need to be revised. The risk assessment strategy that they propose relies on understanding genomic regions involved in food allergy and the role of the gastrointestinal tract.

RESPONSE: We appreciate the positive comments on our manuscript.

The authors identify an important unmet need. They tease the reader with some very interesting ideas but then they do not follow these up with a well-rounded discussion of the opportunities. I give below some examples of issues that would benefit from additional discussion:

1. There is a critical mass of literature that has emerged in recent years on immunogenicity assessments. These include, unwanted immune responses to proteins used in therapies, designing more efficacious vaccines for infectious diseases and cancer, understanding and circumventing autoimmunity etc. The authors correctly recognize that the route of entry of the allergen and the type of immune response that is of concern are important considerations and must be included in a risk assessment strategy. The authors clearly state that for novel food proteins the route of delivery is the GI track and that the IgE response is of concern. It would be useful to then use a visual aid to describe how this information could be incorporated into the assessment strategy. For instance:

* How would you expect the antigen to be presented? You mention HLA - given the route of entry and location of the antigen would you expect the involvement of HLA Class I or II or both?

RESPONSE: The human major histocompatibility complex (MHC) region encodes the human leukocyte antigen (HLA) genes, among which HLA class II molecules (HLA-DR, -DQ and -DP) have especially been reported to be involved in food allergy responses (Khor et al., Scientific 
Reports 8:1069 (2018)). With the advancement of methods for genotyping, genome-wide association studies (GWASs) have been used to identify susceptibility genes for food allergic reactions. In this context, several genome-wide association studies (GWAS) focused on populations from different geographical area have identified food allergy-specific loci in the HLA class II region. Concretely, a GWAS in food allergy (milk, egg, peanut) using a large cohort $(2,759)$ of US children and their parents identified peanut allergy-specific loci in the HLA-DR and $D Q$ regions. Both affected significantly DNA methylation in the HLA-DRB1 and HLA-DQB1 genes, however epigenetic factors can mediate the risk of peanut allergy (Hong et al., Nature Communications 6:6304 (2015)). The association between peanut allergy and variants of the HLA-DRB1 gene has been recently corroborated using a cohort of 221 Australian children (Martino et al., Clinical and Experimental Allergy 47:217 (2017)). Similarly, another GWAS performed on 11,011 Japanese women has identified shrimp-and peach allergy-specific loci in the HLA region encompassing six HLA class II genes (HLA-DQA1, HLA-DRB5, HLA-DRB1, HLADQB1, HLA-DQA2, HLA-DRA) (Khor et al., Scientific Reports 8:1069 (2018)). Finally, a GWAS on oral food challenge-proven food allergic children in Germany has identified five genomic regions associated with IgE-food allergy (Marenholz et al., Nature Communications 8:1056 (2017)): of these, the HLA-DQB1 locus was confirmed to be associated to peanut allergy, whereas the other four loci (the clade B serpin (SERPINB) gene cluster, the cytokine gene cluster, the filaggrin gene and the C11orf30/LRRC32 locus) were associated with a general increase of risk for food allergy. Remarkably, all these loci are involved in either the regulation of the immunological response, or the maintenance of the epithelial barrier, emphasizing the fundamental role of both mechanisms in food allergy regulation. All these studies have been conveniently cited in our manuscript (from reference 7 to 10) and, therefore, we believe that it contains the most updated information on advances to identify HLA types related to food allergy. Nevertheless, as mentioned above, by considering the large heterogeneity of proteins described as IgE allergens and given that the vastly majority of genetic determinants of food allergy remain to be identified, at this moment, we cannot give a reliable answer to the reviewer based on the scientific evidence gathered so far.

* If the primary concern is an IgE response; a brief statement about Th1 vs. Th2 responses and how would this fit into the future risk-assessment algorithms you envision?

RESPONSE: We are not clear about this comment from the reviewer. It is well known that a Th2 response plays a determinant role in IgE mediated adverse reactions.. In any case, we 
would like to highlight that the topic of this paper is not only IgE-mediated but also non-IgEmediated adverse immune reactions (e.g. celiac disease).

* There are pathologies associated with both IgE and non-IgE mediated immune responses. Would the workflow incorporate this information? How would it affect the subsequent assessments (e.g. the HLA Classes assessed etc.)?

RESPONSE: In our manuscript we state that the current understanding of the pathogenesis of non-IgE-mediated diseases other than celiac disease is very limited, and additional research to provide a reliable framework for risk assessment is needed. That is why we focus on celiac disease and IgE-food allergic reactions. On top of that, Figure 1 has been now reshuffled to better streamline the workflow (similarities and differences) between celiac disease and IgEmediated adverse reactions. The Figure caption has also been extended in order to provide more information about the workflow to be followed.

2. The authors should elaborate a little about the approach for Celiac Disease (in reference 5) that seems to have informed their thinking and how this could be the basis for a more generalized risk assessment strategy. For instance, reference 5 in your manuscript states; "T cells respond to antigenic peptides bound to an HLA-molecule. In the case of $C D$, it concerns gluten-derived peptides bound to either HLA-DQ2 or -DQ8. However, it is well known that such T cells can also respond to peptides in which one or more amino acids are replaced. This is the basis, for example, for T-cell cross-reactivity towards gluten peptides and homologous, but not identical, peptides derived from barley and rye." Please discuss how you would propose to assess the novel/foreign transgene:

RESPONSE: We are a bit confused here because the reviewer makes reference to some particular findings described in reference 5 (EFSA guidance published in 2017) that, although related, are out of the scope of the submitted manuscript. In any case, we kindly refer him/her to the guidance which thoroughly describes how the novel protein can be assessed with several examples dealing with non-identical peptides.

* Would you advocate a tiered approach? A broad based in silico approach for high affinity binding of transgene to a diverse cross section of HLA alleles followed by in vitro or ex vivo assays? How would you set a cut-point for "high affinity"? 
RESPONSE: If the reviewer meant celiac disease, the tiered approach is described in Figure I which is included in the Box 1 and it is also clarified in the legend of the Figure 1. If the referee meant IgE-mediated reactions, please see above responses to reviewers 1 and 2 about HLA alleles.

* How would you select the HLA alleles in your screen? Would you consider/weight the alleles based on their frequency in the population? Would you perform analyses for different ethnic and/geographic populations based on HLA alleles that occur at higher frequencies specific populations?

RESPONSE: If the referee meant celiac disease, the current knowledge available on HLA-DQ structures and on the binding recognition sites for T-cell activation, allows defining accurate strategies for assessing the potential CD capacity of proteins as it is indicated in the Box 1. If the reviewer meant IgE-mediated adverse reactions, please see above response to reviewer 1 dealing with personalized risk assessment and also to those to reviewer 2 about HLAphenotyping.

3. The authors may consider including the potential use of new technologies such as the socalled MHC Associated Peptide Proteomics (MAPPs) assay to directly interrogate the MHC proteins from volunteers for the presence of transgene peptides.

RESPONSE: We thank the reviewer for his/her suggestion which has been incorporated in the new Figure 1 as a potential tool.

\section{REVIEWER \#3:}

\section{General comments}

The authors propose a framework for improving prediction of the risk of IgE-mediated responses to ingested food proteins, based on the success of an analogous approach to predicting the risk that a protein will stimulate a response in individuals susceptible to coeliac disease. The approach is based on a combination of in silico and in vitro approaches, including in vitro simulated gastrointestinal digestion. It follows logically from a consideration of the separate elements and their roles. It emphasises more physiological conditions for in vitro digestion and advocates a thorough analysis of the peptides formed, starting with their physicochemical characterisation, but then moving onto investigation of their potential 
participation in relevant immune responses (HLA-binding, T-cell stimulation), if necessary. The conclusions identify a number of important gaps in data and knowledge which need to be addressed in future research.

RESPONSE: We appreciate the positive comments on our manuscript.

The potential advantages of the more physiological digestion protocol(s) in terms of producing better characterised peptides that offer better possibilities for predicting immunogenicity are described in rather more detail than how the subsequent analysis might be effectively undertaken. A particular concern is the statement that the simulated digestion could define 9amino acid peptides as the basis for the subsequent analysis. While the length of the fragment that actually participates directly in the binding within the MHC II is 9 amino acids long), it is recognised that the flanking regions contribute to binding affinity etc (see Meydan et al 2013). Thus using 9-mers as the starting point for further analysis could be misleading and overpredictive, particularly given the well-documented promiscuity of peptide binding to MHC II. The need for validating this and other criteria is indeed mentioned in the conclusions.

The recent paper of Schneidman-Duhovny et al (2018) may also be of interest in relation to this manuscript.

RESPONSE: We acknowledge the two references provided by the reviewer. The size cut-off is scientifically justified by the minimum peptide length necessary for HLA binding (as determined for celiac disease) and T cell activation. For consistency reasons, an overall cut-off value of 9 amino acids was included in the original EFSA guidance document and, consequently, proposed for the assessment of the peptide profile resulting for the in vitro digestion model in our manuscript. However, in the EFSA guidance document is mentioned that the minimal size for IgE-mediated allergic reactions and antibody cross-linking is less clear, and might be more than 9 amino acids. In fact, IgE reactivity and formation of immune complexes may depend on the proximity and number of IgE epitopes (Huby et al., Toxicological Science 55:235 (2000); Gieras et al., Journal of Allergy and Clinical Immunology 137:1557 (2016)), which is in line with reviewer's comment. Therefore, in contrast to T-cell epitopes, the minimum size of peptides which might act as B-cell receptor epitopes and cause IgE cross-linking is less clear, and will require the presence of multiple epitopes (at least two) which can only be accommodated in peptides greater than 9 amino acids in length (Harwood and Batista, Annual Review of Immunology 28:185 (2010); Handlogten et al., Nature Chemical Biology 9:789 (2013)). Nevertheless, it should bear in mind that not only length but also persistence and abundance of 
the fragments in a digest are important aspects to be considered. This is now clearly indicated in the new version of the Figure 1. In this context, it is reasonable to consider that the smaller the fragments are after in vitro digestion the lower the risk of adverse reactions is.

To sum up, it is stated in our manuscript that the cut-off value of 9 amino acids in length is taking into account the minimum amino acid size able to activate T-cells and that this value needs to be verified together with the criteria of persistence and abundance for the identification of digestion fragments as relevant for the risk assessment.

Specific comments

Lines 29-31: it would be useful if the limitations of the current, classical risk assessment approach were summarized

RESPONSE: Taking into account: i) the space constraints of this format; ii) that the comment is referred to the first section of the manuscript dealing with general concepts; and iii) that we provide a useful resource as a footnote (https://doi.org/10.1016/i.toxlet.2018.06.722) where the reader can find more detailed information, we think it is not appropriate to give more details due to space constrains.

Line 59: for greater clarity suggest "regarding their potential to cause CD"

RESPONSE: The text has been clarified following editor's suggestion.

Lines 66-69: The statement is rather generic and, to my knowledge it has not been argued against either. It might be more specific to say "recognition of proteins is dependent on genetic parameters (e.g. MHC haplotypes), but they are difficult to define and use in a risk assessment setting. (Reference (6) indeed illustrates this point).

RESPONSE: we suggest keeping the sentence as it was originally formulated. The statement is indeed rather generic and in style for Forum articles which should be written in a more "journalistic"style.

Line 101: Suggest: persistence/transience rather than persistency/transiency

RESPONSE: Changes done as requested. 
Line 103: see under general comment

RESPONSE: Please, see above our reply to the general comment.

Line 122: suggest: "..hazardous for the remaining $1 \%$ susceptible individuals.."

RESPONSE: The text has been changed as suggested.

Line 127: Missing "is" between "as" and "the"

RESPONSE: The text has been amended as the reviewer and editor suggested. 


\section{Figure}

\section{Oral exposure} to novel food proteins
Physiological in vitro digestion model(s) to better frame protein - GI tract interaction
If resulting fragments are

$\geq 9$ amino acids and

persistent

and abundant
Relevant risk assessment tools

Celiac disease

Optimized (see Box 1)
Potential hazards for immune-mediated adverse reactions

\section{IgE-mediated reactions}

In use: sequence identity (for predicting cross-reactivity).

To be developed: validated bioinformatic approaches (consideration of amino acid physico-chemical properties, etc.).

\begin{tabular}{|l|l|}
\hline In vitro & Optimized (see Box 1) \\
\hline
\end{tabular}

In use: pepsin-resistance test and specific human sera screen (for predicting cross-reactivity).

To be developed: validated i) digestion models (considering additional steps with small intestinal brush border enzymes); ii) gut epithelial cell-based approaches (antigen-presenting cells, MHC-associated peptide proteomics-MAPPs, etc.).

\begin{tabular}{|l|l|}
\hline In vivo & Not foreseen \\
\hline
\end{tabular}

Oral challenges in allergic humans.

To be developed: i) HLA-phenotyping in humans (key for more effective MAPPs assays); ii) validated animal models (relevant for sensitization and for the establishment of doseresponse relationship). 
Knowledge on the protein (exposure and source)
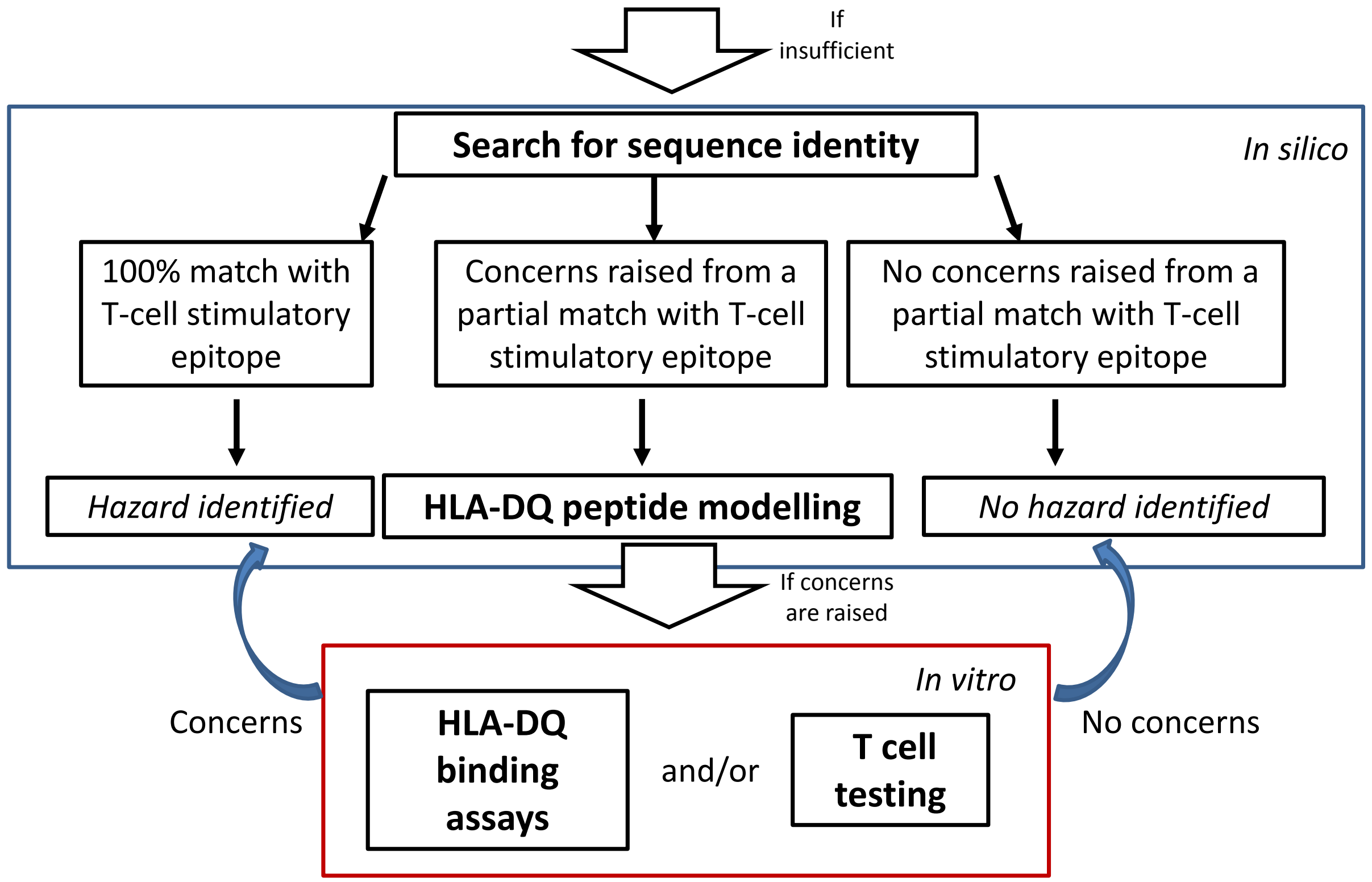
Click here to access/download Manuscript - Editors Comments
Manuscript_edited_revised version_2.docx 